\title{
Esophageal Bolus Domain Pressure and Peristalsis Associated With Experimental Induction of Esophagogastric Junction Outflow Obstruction
}

\author{
Wei-Yi Lei, ${ }^{1}$ Taher Omari, ${ }^{2}$ Tso-Tsai Liu, ${ }^{1}$ Ming-Wun Wong, ${ }^{1}$ Jui-Sheng Hung, ${ }^{1}$ Chih-Hsun Yi, ${ }^{1}$ Shu-Wei Liang, ${ }^{1}$ Charles Cock, ${ }^{2}$ and \\ Chien-Lin Chen ${ }^{1 *}$ \\ ${ }^{I}$ Department of Medicine, Hualien Tzu Chi Hospital, Buddhist Tzu Chi Medical Foundation and Tzu Chi University, Hualien, Taiwan; and ${ }^{2}$ College \\ of Medicine \& Public Health Flinders University, Adelaide, SA, Australia
}

\section{Background/Aims}

Intrabolus pressures are important for esophageal bolus transport and may detect obstructed bolus flow. This study measured the effect esophageal outflow obstruction experimentally induce by a leg-lift protocol.

\section{Methods}

Twenty-five gastroesophageal reflux disease patients referred for esophageal manometry and a normal motility diagnosis were included. Supine liquid swallows were tested. Leg-lift protocol generated esophageal outflow obstruction by increasing abdominal pressure. Esophageal pressure topography and intrabolus pressure metrics were calculated. These included, (1) mid-domain bolus distension pressure during esophageal emptying (DPE, $\mathrm{mmHg}$ ) and (2) ramp pressure $(\mathrm{mmHg} / \mathrm{sec})$, generated by compression of the bolus between the peristaltic contraction and esophagogastric junction (EGJ).

\section{Results}

EGJ relaxation pressure was increased by leg-lift from $13(11-17)$ to $19(14-30) \mathrm{mmHg}(P<0.005)$ and distal contractile integral also increased from 1077 (883-1349) to $1620(1268-2072) \mathrm{mmHg} \cdot \mathrm{cm} \cdot \mathrm{sec}(P<0.001)$ as a physiological response to obstruction. All bolus pressures were increased by leg lift; DPE increased from $17(15-20)$ to $27(19-32) \mathrm{mmHg}(P<0.001)$, and ramp pressure increased from $3(1-4)$ to $5(2-9) \mathrm{mmHg} / \mathrm{sec}(P<0.05)$.

\section{Conclusion}

Measuring pressures within the intrabolus domain can quantify changes related to obstruction to outflow and may serve as adjunct measures for confirming a diagnosis EGJ outflow obstruction.

\section{(J Neurogastroenterol Motil 2022;28:62-68)}

\section{Key Words}

Esophagogastric junction; Esophagus; Manometry; Peristalsis; Pressure

Received: September 30, 2020 Revised: None Accepted: January 2, 2021

(a) This is an Open Access article distributed under the terms of the Creative Commons Attribution Non-Commercial License (http://creativecommons. org/licenses/by-nc/4.0) which permits unrestricted non-commercial use, distribution, and reproduction in any medium, provided the original work is properly cited.

*Correspondence: Chien-Lin Chen, MD, PhD Department of Medicine, Hualien Tzu Chi Hospital, Buddhist Tzu Chi Medical Foundation and Tzu Chi University, 707, Section 3, Chung-Yang Road, Hualien 970, Taiwan Tel: +886-3-8561825, Fax: +886-3-8577161, E-mail: harry.clchen@msa.hinet.net 


\section{Introduction}

High-resolution manometry (HRM) and the derivation of esophageal pressure topography (EPT) metrics ${ }^{1,2}$ has enhanced knowledge of esophageal physiology and enabled clinical translation for diagnosis of esophageal motor disorders via the Chicago classification (CC) algorithm (current version 3.0). ${ }^{3}$ The $\mathrm{CC}$ defines lower esophageal sphincter relaxation, with relaxation failure required to diagnose achalasia and esophagogastric junction outflow obstruction (EGJOO). Four-second integrated relaxation pressure (4sIRP) is, however, a complex metric influenced by both the intrinsic relaxation of the lower esophageal sphincter, extrinsic compression by the crural diaphragm and contact artefact generated by luminal impingement onto the catheter. ${ }^{4}$ In the case of EGJOO, concerns have been raised over the clinical significance of high values for 4sIRP leading to a high rate of EGJOO diagnoses and the potential for misdiagnosis and in turn inappropriate treatment. ${ }^{5-9}$

Studies have shown that esophageal intraluminal impedance can determine the phases of luminal bolus distension and how they relate contraction during esophageal bolus transport. Directly linking bolus flow to pressure may reveal mechanisms of symptom generation. ${ }^{10-14} \mathrm{~A}$ range of adjunctive integrated pressure-flow metrics can be derived by combining impedance topography with manometry to better quantify the pressures generated within the intrabolus domain during esophageal bolus transport. ${ }^{15}$ It remains to be determined whether these novel integrated metrics may play a role as adjunct measures to confirm restricted esophageal emptying. In this study we measured the effect of vertical leg-lift, a protocol designed to induce an EGJOO-like experimental scenario by artificially increasing the gastric pressure, thus creating a greater pressure differential against which the esophageal peristalsis must work to enable complete esophageal emptying of a swallow bolus into the stomach. We hypothesized that leg lift would increase bolus domain pressures and delay esophageal emptying with associated physiological changes such as augmentation of contractile forces.

\section{Materials and Methods}

\section{Study Subjects}

Between August 2018 and July 2019, we prospectively enrolled consecutive patients with gastroesophageal reflux disease (GERD) symptoms. Patients diagnosed with normal motility using $\mathrm{CC}$ version 3.0 criteria $^{3}$ were included. The exclusion criteria consisted of: nasolaryngeal esophageal stricture; history of thoracic, esophageal or gastric surgery; and musculoskeletal disorder that would limit the patients' mobility. Patients with dysphagia symptoms were also excluded. The medical records of included patients were reviewed, and demographic information, medications, medical and surgical history were extracted. Any medication that could affect upper gastrointestinal motility was discontinued 2 weeks prior to study. All subjects were asked to complete validated GERD questionnaires and receive endoscopic examination. The presence of mucosal injury in the lower esophagus and hiatal hernia was recorded.

The study protocol was approved by the Research Ethics Committee of the Hualien Tzu Chi Hospital (IRB No. 107-180-A), confirming that the study was conducted in accordance with the ethical guidelines of the Declaration of Helsinki.

\section{High-resolution Impedance Manometry Equipment}

HRM was performed using solid state catheter incorporating impedance (8F, 32 pressure sensors at $1 \mathrm{~cm}$ spacing and 17 impedance segments at $2 \mathrm{~cm}$; Unisensor/Laborie, Attikon, Switzerland). Pressure and impedance data were acquired at $20 \mathrm{~Hz}$ (Solar GI acquisition system; Medical Measurement Systems, Enschede, The Netherlands).

\section{Study Protocol}

Patients fasted overnight. The HRM assembly was passed into the esophagus through the anesthetized nostril (lignocaine spray). The catheter was zeroed to atmospheric pressure and placed with at least 3 distal sensors positioned in the stomach, to record the region from the upper esophageal sphincter (UES) to the proximal stomach. While in the supine position, each patient was given 10 liquid swallows of $5 \mathrm{~mL}$ volume (Standardized Bolus Medium Kit for high-resolution impedance manometry [HRIM], Trisco

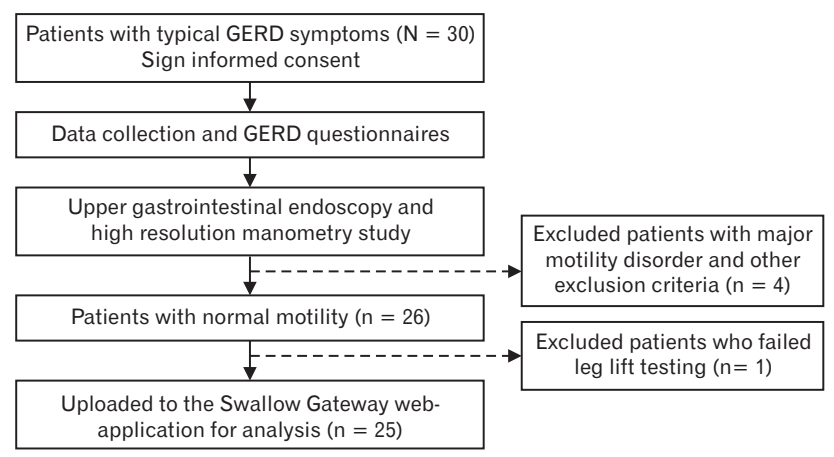

Figure 1. The flowchart of patients enrolled into the study. GERD, gastroesophageal reflux disease. 


\section{A Pressure-flow topography}

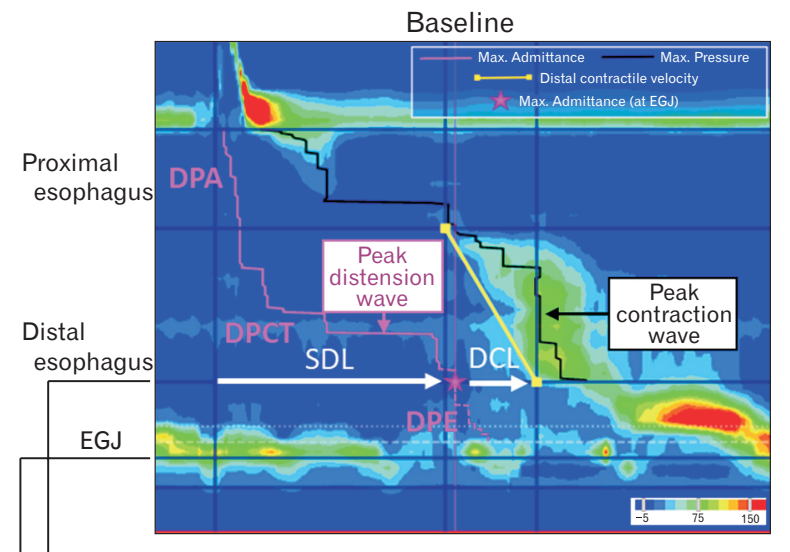

Distension and Pressure distal esophagus

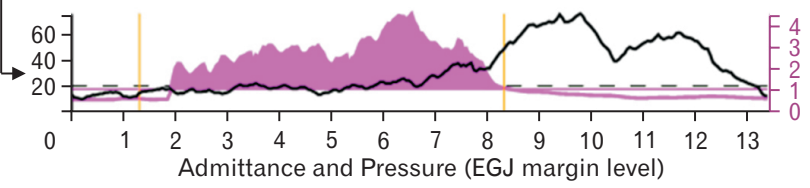

Distension and Pressure EGJ

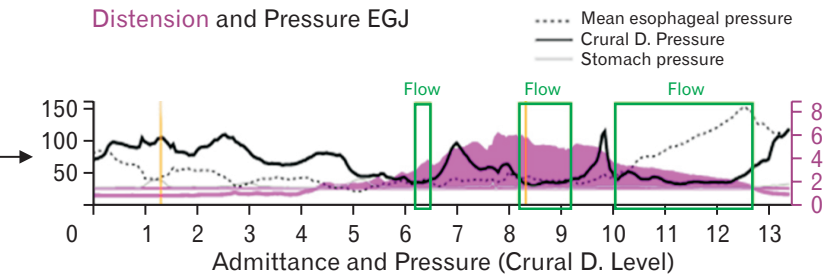

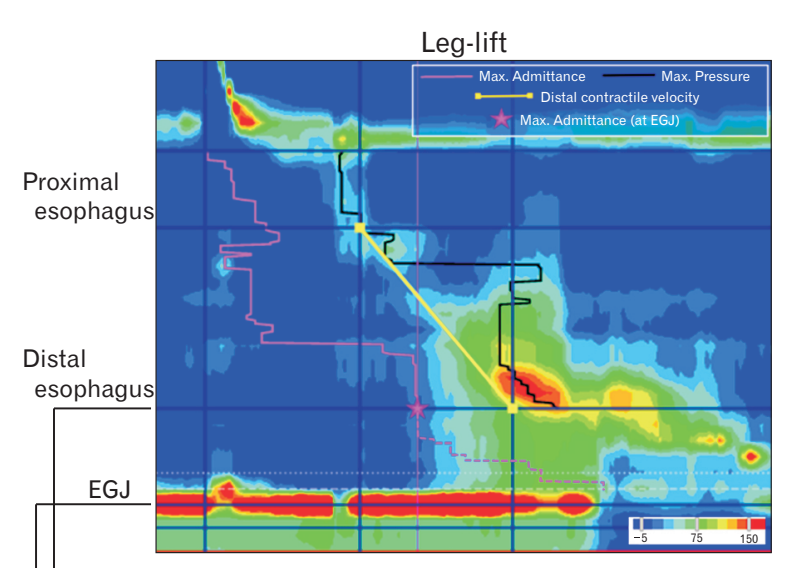

Distension and Pressure distal esophagus
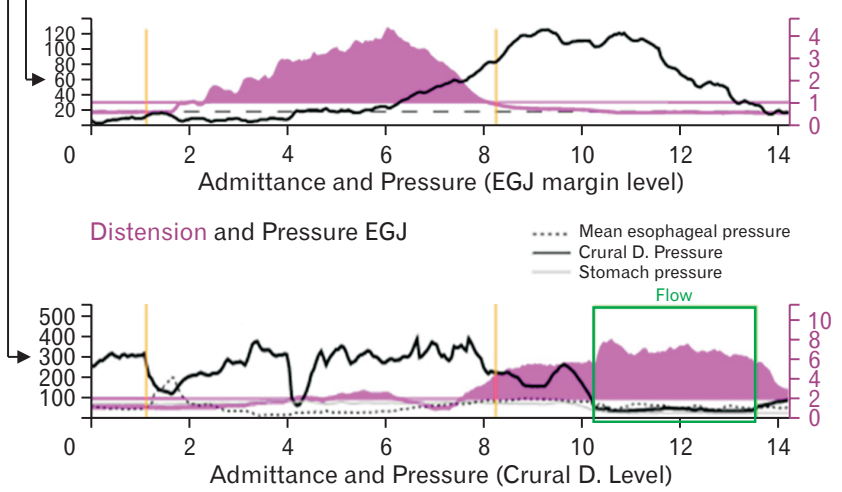

B Integrated pressure-flow metrics

Distension and Pressure distal esophagus

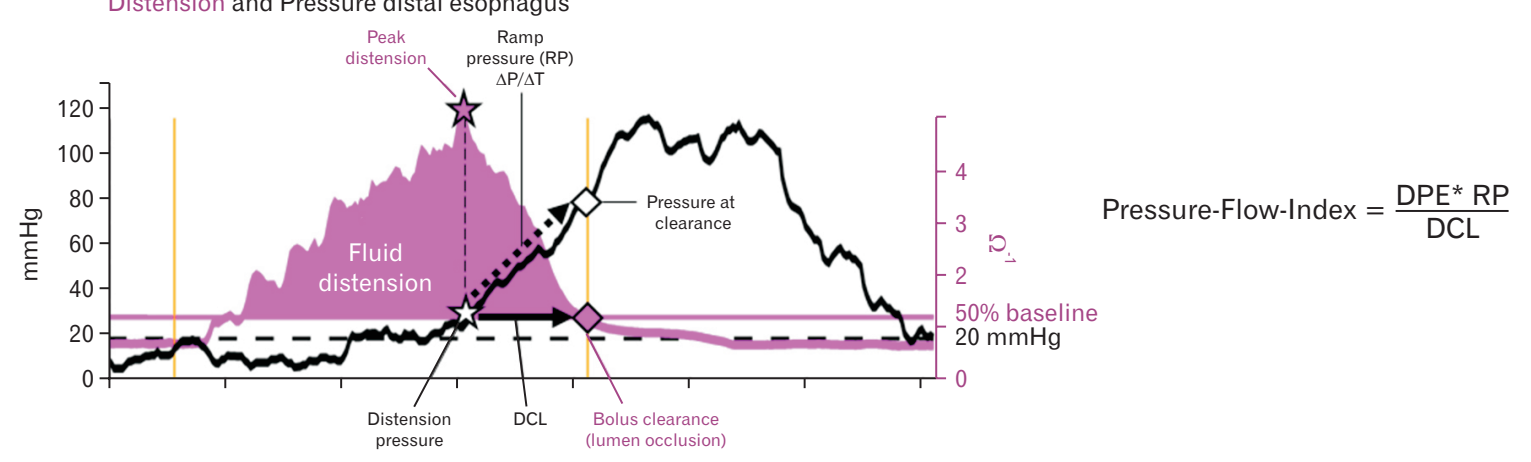

Figure 2. Integrated pressure-flow analysis. (A) Example esophageal pressure topography plots under the 2 experimental conditions. Superimposed lines indicate the time and position of peak bolus distension (thick purple line) and peak esophageal contraction (thick black line). Swallow to distension latency (SDL, sec) and distension to contraction latency (DCL, sec) indicate the time interval from swallow to peak distension and then to lumen occlusive distal contraction. Distension pressure during accommodation (DPA, mmHg), Compartmentalized transport (DPCT, $\mathrm{mmHg}$ ), and esophageal emptying (DPE, $\mathrm{mmHg}$ ) are mean of peak distension pressure values measured in the proximal esophagus, distal esophagus and esophagogastric junction (EGJ) regions. The plots below the topography show the separate distension (pink) and pressure (black) segment profiles recorded during bolus passage at the distal margin of esophageal body peristalsis (upper plot) and at the EGJ midpoint (lower plot). "Flow" indicates the time intervals where the bolus is present above the EGJ and there is also a favorable esophagogastric pressure gradient. The sum of flow events determines the total bolus flow time (BFT, sec). (B) The intra-bolus ramp pressurization $(\mathrm{RP}, \mathrm{mmHg} / \mathrm{sec})$ is determined as the mean gradient of pressure change over time measured from peak distension (star symbols) to bolus clearance (luminal closure; diamond symbol). 
Foods, Pty Ltd, Queensland, Australia) at 30-second intervals. After completion of baseline recording, participants were asked to gently raise both legs by flexing the hip to 40 degrees with the knees in extension for at least 5 seconds. Five $5 \mathrm{~mL}$ single water swallows with leg lift were then administered during HRM through a syringe. Following data acquisition, each de-identified pressureimpedance file was exported as an American Standard Code for Information Interchange (ASCII)-file and uploaded to the Swallow Gateway web-application (Flinders University, Adelaide, Australia) for analysis. ${ }^{16}$ The flowchart of the study is shown in Figure 1.

\section{Data Analysis}

The averages of liquid swallows at baseline and during leg-lift were compared. EPT variables determined were: $4 \mathrm{~s}-\mathrm{IRP}(\mathrm{mmHg})$, proximal contractile integral (PCI, $\mathrm{mmHg} \cdot \mathrm{cm} \cdot \mathrm{sec})$, distal contractile integral (DCI, $\mathrm{mmHg} \cdot \mathrm{cm} \cdot \mathrm{sec})$, contractile front velocity $(\mathrm{CFV}$, $\mathrm{cm} / \mathrm{sec}$ ), distal latency (DL, sec), and largest break size $(\mathrm{cm}){ }^{3}$

The adjunct measures used to quantify the pressures and flowtiming within the intrabolus domain are illustrated in Figure 2. These included 3 mid-domain intrabolus distension pressures (DP) during phases of esophageal bolus transport; ${ }^{17}$ namely DP during accommodation (mean DP in $\mathrm{mmHg}$ from UES to transition zone), compartmentalized transport (mean DP in $\mathrm{mmHg}$ from transition zone to esophagogastric junction [EGJ] proximal margin), and esophageal emptying (DPE, mean DP in $\mathrm{mmHg}$ from EGJ margin to crural diaphragm). Intrabolus ramp pressure (RP, $\mathrm{mmHg} / \mathrm{sec}$ ), was used to quantify pressure increase as the bolus is compressed between the esophageal contraction and the EGJ. Flow timing measures included the swallow to mid-bolus distension latency (sec), mid-bolus distension to contraction latency (DCL, sec). Impedance ratio is a parameter defining bolus clearance. ${ }^{15}$ Three of the measures (DPE, RP, and DCL) were combined to derive the Pressure-Flow Index, an experimental composite score of impeded esophageal transport. ${ }^{15}$ Finally, the period of trans-EGJ bolus emp- tying was estimated based on the method of Lin et $\mathrm{al}^{18}$ by using the impedance, to detect bolus presence, and the pressure to detect flow-permissive pressure gradient intervals (ie, esophageal pressure $>$ crural and gastric pressure) within to the overall period of bolus presence. Bolus flow time (BFT, sec) was defined by the sum of the flow permissive periods. Shorter BFT reduced esophageal emptying. ${ }^{18}$

\section{Statistical Methods}

Statistical tests were performed using IBM SPSS version 22 (IBM, Armonk, NY, USA). Data are presented as either mean (standard deviation) or median (interquartile range). Subject average data for each experimental condition were derived compared using Wilcoxon signed rank test. Significance was accepted at a $P$ value $<0.05$; however, $P$-values of 0.050-0.099 are also shown.

\section{Results}

Twenty-five patients were included (mean age: 42.0 years; $52 \%$ female), 5 had globus sensation with typical GERD symptoms and the remainder reported symptoms of heartburn and/or regurgitation. None had evidence of esophagitis or hiatal hernia. The esophageal acid exposure time during diagnostic $\mathrm{pH}$-monitoring ranged from 2.0-22.5\% (mean: $3.0 \pm 4.6 \% ; \mathrm{n}=2$, AET $>6 \%$ ). All study participants successfully completed the protocol and no adverse events occurred. For 3 studies the 4s-IRP values were not calculable during leg lift protocol due to movement of the catheter leading to a poor intragastric pressure reading.

The effects of the leg lift protocol on EPT metrics are shown in Table 1. Leg lift caused a significant increase 4s-IRP. PCI and DCI also increased, consistent with augmented of peristaltic vigor as a physiological response to resistance. However, the effect on DCI was far more potent. There was no effect of leg lift on any of the remaining EPT variables.

Table 1. Effect of Leg-lift on Esophageal Pressure Topography Variables Generated During Liquid Swallows in Healthy Subjects

\begin{tabular}{lccc}
\hline \multicolumn{1}{c}{ Esophageal pressure topography variables } & Before leg-lift & During leg-lift & Wilcoxon $t$ \\
\hline Proximal contractile integral $(\mathrm{mmHg} \cdot \mathrm{cm} \cdot \mathrm{sec})$ & $303(160-383)$ & $303(245-453)^{\mathrm{a}}$ & 2.193 \\
Distal contractile integral $(\mathrm{mmHg} \cdot \mathrm{cm} \cdot \mathrm{sec})$ & $1077(883-1349)$ & $1620(1268-2072)^{\mathrm{b}}$ & $6.7(6.3-7.2)$ \\
Distal latency $(\mathrm{sec})$ & $6.8(6.1-7.3)$ & $4.3(3.4-4.6)$ & - \\
Contractile front velocity $(\mathrm{cm} / \mathrm{sec})$ & $4.0(3.4-4.7)$ & $0.0(0.0-0.0)$ & - \\
Largest break $(\mathrm{cm})$ & $0.0(0.0-0.0)$ & $19(14-30)^{\mathrm{c}}$ & 3.165 \\
4s-IRP $(\mathrm{mmHg})$ & $13(11-17)$ & & \\
\hline
\end{tabular}

4s-IRP, 4-second integrated relaxation pressure.

${ }^{\mathrm{a}, \mathrm{b}, \mathrm{c}}$ Indicate significantly different by Wilcoxon signed rank test $\left({ }^{\mathrm{a}} \mathrm{P}<0.05,{ }^{\mathrm{b}} \mathrm{P}<0.001,{ }^{\mathrm{c}} \mathrm{P}<0.005\right)$; standardized $t$ and $P$-values are shown when the $P$-value was $<0.1$. Data are expressed as median (interquartile range). 
Table 2. Effect of Leg Lift on Integrated Pressure-Flow Variables Generated During Liquid Swallows in Healthy Subjects

\begin{tabular}{|c|c|c|c|}
\hline Integrated pressure-flow variables & Before leg-lift & During leg-lift & Wilcoxon $t$ \\
\hline \multicolumn{4}{|l|}{ Bolus clearance } \\
\hline Impedance ratio & $0.34(0.27-0.54)$ & $0.33(0.29-0.58)$ & - \\
\hline Pressure-Flow-Index & $25(12-49)$ & $54(21-246)^{c}$ & 3.780 \\
\hline \multicolumn{4}{|l|}{ Intrabolus DP } \\
\hline $\mathrm{DP}$ during accommodation $(\mathrm{mmHg})$ & $10(8-15)$ & $11(7-15)$ & - \\
\hline DP compartmentalized transport $(\mathrm{mmHg})$ & $14(11-16)$ & $17(13-21)^{b}$ & 3.296 \\
\hline DP esophageal emptying (mmHg) & $17(15-20)$ & $27(19-32)^{c}$ & 4.345 \\
\hline Ramp pressurization $(\mathrm{mmHg} / \mathrm{sec})$ & $3(1-4)$ & $5(2-9)^{a}$ & 2.301 \\
\hline \multicolumn{4}{|l|}{ Flow timing } \\
\hline Swallow to distension latency (sec) & $4.6(3.7-5.1)$ & $4.6(3.7-5.5)^{\mathrm{a}}$ & 1.870 \\
\hline Distension to contraction latency (sec) & $2.3(1.9-3.0)$ & $2.2(1.3-2.5)^{\mathrm{a}}$ & -2.247 \\
\hline Bolus flow time (sec) & $2.9(1.9-3.5)$ & $2.8(1.6-3.3)$ & - \\
\hline
\end{tabular}

DP, distention pressure.

${ }_{\mathrm{a}, \mathrm{b}, \mathrm{c}}$ Indicate significantly different by Wilcoxon signed rank test $\left({ }^{\mathrm{a}} \mathrm{P}<0.05,{ }^{\mathrm{b}} \mathrm{P}<0.005,{ }^{\mathrm{c}} \mathrm{P}<0.001\right)$; standardized $t$ and $P$-values are shown when the $P$-value was $<0.1$. Data are expressed as median (inter-quartile rang).

The effects of the leg lift protocol on integrated pressure-flow metrics are shown in Table 2. The esophageal impedance ratio and BFT were not significantly altered by leg lift, suggesting that the experimentally induced obstruction did not lead to failure of bolus transport and/or esophageal emptying in these participants with normal motility and the ability to augment contractility in response to leg lift (increased DCI previously described). The mid-bolus distension pressures increased in response to leg lift with the effect being most significant in the distal esophagus during the esophageal emptying phase (DPE). RP in the distal esophagus was also significantly increased. The DCL was reduced, suggesting that the mid bolus distension was shifted closure in time to the contraction front. Overall, the Pressure-Flow-Index score was significantly increased by leg lift (25 [12-49] to 54 [21-246], $P<0.001)$.

\section{Discussion}

In this study esophageal HRIM examined the response to leg lift, designed to induce esophageal outflow obstruction through an increase in intra-gastric pressure. The main finding was that, in participants with normal esophageal body motility, bolus swallows performed during leg lift showed an increase in the mid-bolus distension pressure and an increase in contractile vigor. These effects were most potent in the distal esophageal body.

Classic research papers have described in detail the concept of there being 2 pressure domains, firstly, within the bolus and then, secondly, within the contractile segment devoid of bolus. ${ }^{19}$ However, the importance of measuring and understanding intrabolus forces and flow timing characteristics for diagnostic purposes continues to be under-recognized, primary focus being the classification of contractility patterns. The current study examined the adjunct value of combined esophageal impedance and pressure measurement to quantify forces within the moving bolus that were only previously attainable via esophageal video-mano-fluorography and frame by frame analysis. Studies have now confirmed the veracity of the method of combing and analyzing intraluminal impedance with pressure to map the trajectory of the bolus domain over space and time and to measure the forces within. Using this pressure-flowanalysis approach it is possible to derive several novel measures of flow timing, luminal area, bolus presence, distension pressure, RPs, and clearance.

The leg lift protocol is an experimental provocative maneuver that we are trialing in conjunction with routine diagnostic esophageal HRM. Whilst we report data from GERD patients, rather than asymptomatic controls, the patients included had normal baseline motility, no hiatus hernia, or esophagitis. Therefore, it was felt that they would provide a suitable cohort to examine the biomechanical changes in the esophagus induced when bolus emptying into the stomach is artificially impeded. Past studies show that abdominal compression causes outflow resistance indicated by an increase in intrabolus pressure. In our hands, the simple to perform leg lift appears to have a larger impact on pressure-flow metrics than we observed using extrinsic abdominal compression utilizing a gastric binder. ${ }^{20}$ The effect of leg lift on contractile pressures mirrors that seen with other paradigms and is the normal physiological response. As demonstrated by Dodds et $\mathrm{al}^{21}{ }^{21}$ passive distension by 
the moving bolus stimulates and augments contractility producing higher peak pressure and a longer contraction phase (both of which increase the DCI metric). Further supra-physiological increases in emptying resistance lead to greater passive distension of the esophageal lumen that further stimulates intrinsic neuro-mechanical responses via sensory afferents and polarized enteric nervous system circuits, ${ }^{22}$ and changes the pre-load, and after-load muscle tension. ${ }^{23}$ Together these factors drive the higher contractile pressures that are need to overcome obstruction, thus preventing transport failure associated with retrograde bolus escape due to the intrabolus pressure remaining below the capacity of the circular muscle to achieve lumen occlusion at the bolus tail. ${ }^{19}$ As these mechanisms were intact in the studied patients with normal motility, leg lift did not lead to failure of bolus transport (ie, IR and BFT were not altered). Further studies are needed to determine the rate of transport failure in patients with, for example, ineffective esophageal motility.

In conclusion, novel measurement and analysis methods allow comprehensive characterization of pressures within the intrabolus domain without the need for simultaneous radiology. These pressures were augmented along with the $4 \mathrm{~s}$-IRP during experimental outflow obstruction produced by leg lift. Further studies could explore the potential adjunct value of such measures for confirming clinically relevant EGJOO in symptomatic patients.

Financial support: This study was supported by a grant, TCRD108-28, from Hualien Tzu Chi Hospital, Buddhist Tzu Chi Medical Foundation, Hualien, Taiwan. Taher Omari holds a National Health and Medical Research Council Senior Research Fellowship. The development of the swallowgateway.com website was supported by grants from the College of Medicine and Public Health, Flinders University. The other authors report no external funding for this manuscript.

Conflicts of interest: Taher Omari holds inventorship of the patent family that covers some of the analytical methods described. The Swallow Gateway web application is owned by Flinders University. All other authors report no conflict of interest.

Author contributions: Study concept and design, acquisition, analysis, or interpretation of data: Chien-Lin Chen and Taher Omari; acquisition, analysis, or interpretation of data, and drafting of the manuscript: Chien-Lin Chen, Taher Omari, Charles Cock, Wei-Yi Lei, Tso-Tsai Liu, Ming-Wun Wong, Jui-Sheng Hung, Chih-Hsun Yi, and Shu-Wei Liang; critical revision of the manuscript for important intellectual content: all authors; and administrative, technical, or material support, and study supervision: Chien-
Lin Chen.

\section{References}

1. Pandolfino JE, Kwiatek MA, Nealis T, Bulsiewicz W, Post J, Kahrilas PJ. Achalasia: a new clinically relevant classification by high-resolution manometry, Gastroenterology 2008;135:1526-1533.

2. Kahrilas PJ. Esophageal motor disorders in terms of high-resolution esophageal pressure topography: what has changed? Am J Gastroenterol 2010;105:981-987.

3. Kahrilas PJ, Bredenoord AJ, Fox M, et al. The Chicago Classification of esophageal motility disorders, v3.0. Neurogastroenterol Motil 2015;27:160-174.

4. Babaei A, Szabo A, Yorio SD, Massey BT. Pressure exposure and catheter impingement affect the recorded pressure in the Manoscan $360^{\mathrm{TM}}$ system. Neurogastroenterol Motil 2018;30:e13329.

5. van Hoeij FB, Smout AJ, Bredenoord AJ. Characterization of idiopathic esophagogastric junction outflow obstruction. Neurogastroenterol Motil 2015;27:1310-1316

6. Liu A, Woo M, Nasser Y, et al. Esophagogastric junction outflow obstruction on manometry: outcomes and lack of benefit from CT and EUS. Neurogastroenterol Motil 2019;31:e13712.

7. Schupack D, Katzka DA, Geno DM, Ravi K. The clinical significance of esophagogastric junction outflow obstruction and hypercontractile esophagus in high resolution esophageal manometry. Neurogastroenterol Motil 2017;29:1-9.

8. Okeke FC, Raja S, Lynch KL, et al. What is the clinical significance of esophagogastric junction outflow obstruction? evaluation of 60 patients at a tertiary referral center. Neurogastroenterol Motil 2017;29:e13061.

9. Beveridge CA, Falk GW, Ahuja NK, Yang YX, Metz DC, Lynch KL. Low yield of cross-sectional imaging in patients with esophagogastric junction outflow obstruction. Clin Gastroenterol Hepatol 2020;18:16431644.

10. Leibbrandt RE, Dinning PG, Costa M, et al. Characterization of esophageal physiology using mechanical state analysis. Front Syst Neurosci 2016;10:10.

11. Kim JH, Mittal RK, Patel N, Ledgerwood M, Bhargava V. Esophageal distension during bolus transport: can it be detected by intraluminal impedance recordings? Neurogastroenterol Motil 2014;26:1122-1130.

12. Zifan A, Ledgerwood-Lee M, Mittal RK. Measurement of peak esophageal luminal cross-sectional area utilizing nadir intraluminal impedance. Neurogastroenterol Motil 2015;27:971-980.

13. Zifan A, Song HJ, Youn YH, Qiu X, Ledgerwood-Lee M, Mittal RK. Topographical plots of esophageal distension and contraction: effects of posture on esophageal peristalsis and bolus transport. Am J Physiol Gastrointest Liver Physiol 2019;316:G519-G526.

14. Mittal RK, Muta K, Ledgerwood-Lee M, Zifan A. Relationship between distension-contraction waveforms during esophageal peristalsis: effect of bolus volume, viscosity, and posture. Am J Physiol Gastrointest Liver Physiol 2020;319:G454-G461.

15. Omari T, Tack J, Rommel N. Impedance as an adjunct to manometric 
testing to investigate symptoms of dysphagia: what it has failed to do and what it may tell us in the future. United European Gastroenterol J 2014;2:355-366.

16. Swallow Gateway high resolution manometry. Available from URL: https://swallowgateway.com/ (accessed 21 Dec 2021).

17. Lin Z, Yim B, Gawron A, Imam H, Kahrilas PJ, Pandolfino JE. The four phases of esophageal bolus transit defined by high-resolution impedance manometry and fluoroscopy. Am J Physiol Gastrointest Liver Physiol 2014;307:G437-G444.

18. Lin Z, Imam H, Nicodème F, et al. Flow time through esophagogastric junction derived during high-resolution impedance-manometry studies: a novel parameter for assessing esophageal bolus transit. Am J Physiol Gastrointest Liver Physiol 2014;307:G158-G163.

19. Ren J, Massey BT, Dodds WJ, et al. Determinants of intrabolus pressure during esophageal peristaltic bolus transport. Am J Physiol 1993;264(3 pt 1):G407-G413.

20. Brink GJ, Lei WY, Omari TI, et al. Physiological augmentation of esophageal distension pressure and peristalsis during conditions of increased esophageal emptying resistance. Neurogastroenterol Motil 2018;30:e13225.

21. Dodds WJ, Hogan WJ, Reid DP, Stewart ET, Arndorfer RC. A comparison between primary esophageal peristalsis following wet and dry swallows. J Appl Physiol 1973;35:851-857.

22. Goyal RK, Paterson WG. Esophageal motility. In: Wood JD, ed. Handbook of physiology: the gastrointestinal system. Washington, DC: American Physiological Society 1989:865-908.

23. Cohen S, Green F. The mechanics of esophageal muscle contraction. Evidence of an inotropic effect of gastrin. J Clin Invest 1973;52:20292040 . 